\title{
DIMENSIONES EXPLICATIVAS Y RESULTADOS A PARTIR DE VARIABLES SOCIO-DEMOGRÁFICAS Y SOCIO-LABORALES EN LOS ESTILOS DE LIDERAZGO
}

\author{
EXPLANATORY DIMENSIONS AND RESULTS FROM SOCIO-DEMOGRAPHIC \\ AND SOCIO-LABORAL VARIABLES IN LEADERSHIP STYLES \\ gabriela.alava@ucuenca.edu.ec lucia.dominguezv@ucuenca.edu.ec francisco.frances@ua.es
}

\section{Forma sugerida de citar:}

Álava Atiencie, Gabriela [et. al.]< (2016). Dimensiones explicativas y resultados a partir de variables socio-demográficas y socio-laborales en los estilos de liderazgo. Revista Economía y Política, Año XII, No. 24. pp. 67-91

Fecha de recepción: 18 de octubre de $2016 \quad$ Fecha de aceptación: 30 de marzo de 2017

\section{RESUMEN}

El objetivo del presente trabajo fue examinar la influencia de las variables socio-demográficas y sociolaborales en los estilos de liderazgo de diversos líderes ecuatorianos de sectores comunitarios, privados y públicos; así como identificar las dimensiones de los estilos de liderazgo que inciden significativamente en las variables de resultado: eficiencia, eficacia y satisfacción. A partir de la aplicación empírica y de los análisis realizados mediante las técnicas estadísticas Anova y análisis de regresión y correlación los resultados muestran la preeminencia explicativa de variables sociodemográficas (especialmente la edad y el nivel educativo) sobre aquellas propias del contexto socio-laboral en el desarrollo de los estilos de liderazgo, así como la importancia de los componentes propios del liderazgo transformacional en la comprensión de las variables de resultado esperadas en las personas que ejercen roles de liderazgo.

PALABRAS CLAVES: Estilos de liderazgo, LaissezFaire, edad, nivel educación y dedicación laboral.

\section{ABSTRACT}

The aim of this study was to examine the influence of socio-demographic and socio-labor variables in the leadership styles of various Ecuadorian leaders from community private and public sectors; besides identify the dimensions of leadership styles that significantly affect the outcome variables: efficiency, effectiveness and satisfaction. From the empirical application carried out and the analyzes performed using statistical techniques like Anova and regression analysis and correlation results show the explanatory preeminence of socio demographic variables (especially age and educational level) over the specific socio-labor variables in the development of leadership styles, as well as the importance of the own components of transformational leadership in the comprehension of the expected result outcomes variables in people who exercise leadership roles.

KEYWORDS: Leadership Styles: Transformational, Transactional, Laissez-Faire, socio-demographic and socio-labor variables.

\section{JEL: J24, M10}

1 Ingeniera Comercial por la Universidad de Cuenca, Ecuador. Magister en Gerencia y Liderazgo Educacional. Docente titular e investigadora de la Facultad de Ciencias Económicas y Administrativas de la Universidad de Cuenca, Ecuador.

2 Economista por la Universidad de Cuenca, Ecuador. Máster en Administración de Empresas. Docente titular e investigadora de la Facultad de Ciencias Económicas y Administrativas de la Universidad de Cuenca, Ecuador. Actual Subdecana de la Facultad.

3 Doctor en Sociología. Docente e investigador de la Universidad de Alicante, España. 


\section{Introducción: Estilos de Liderazgo y tendencias de aplicación}

Todo tipo de organización, independientemente de su estructura, de actualmente afrontar múltiples desafíos, frente a las exigencias del propio entorno tales como los altos niveles de competencia, la internacionalización económica y a la presencia de nuevas tecnologías, por lo que es preciso que al interior de ella se genere un clima de confianza que motive un esfuerzo integrado, y donde las personas que ejercen el liderazgo apliquen sus capacidades para alcanzar así los objetivos y las metas organizacionales, a través de procesos renovadores e innovadores continuos.

Según Koontz, H., \& Weihrich (1990) los estudios sobre el liderazgo realizados antes de la década de los años cincuenta del siglo pasado solo representaron intentos para identificar las características que poseían los lideres, primando en los resultados la teoría del "gran hombre" en el sentido de que "los lideres nacen y no se hacen". Dicha teoría con el pasar de los años perdió fuerza, cuando la escuela conductista de la Psicología minimizó el componente genético en los rasgos de liderazgo. Parece ser que hoy en día existe un consenso en pensar el liderazgo como aquel que involucra un proceso de influencia. Pero a partir de ese mínimo sustrato de consenso surgen las discrepancias en relación al resto de sus componentes: si el liderazgo es o no coercitivo, si es de naturaleza finalista o relacional, o la identificación de los logros al optar por un estilo concreto, a partir de la evaluación de los resultados que puedan generar para la organización.

En este sentido, a partir de las principales aportaciones teóricas (Yukl, 2010; Chiavenato, 2000; Koontz \& Weihrich, 1990) y con el objeto de alcanzar cierta clarificación conceptual, en este texto entenderemos el liderazgo un "proceso de influencia interpersonal para entender y estar de acuerdo sobre lo que hay que hacer y cómo hacerlo, y el proceso de facilitación de los esfuerzos individuales y colectivos, en el marco de una comunicación asertiva para lograr objetivos comunes". La definición señalada nos lleva a pensar en el liderazgo efectivo como un proceso de gestión del cambio organizacional basado sobre todo en los principios de aprovechamiento del entorno y la anticipación de los cambios. La gestión del cambio organizacional por lo tanto no consiste en implantar nuevos modelos de gestión sino en desarrollar una capacidad de adaptación con un gran sentido de comunicación, flexibilidad, compromiso y responsabilidad (Pascale, 1990).

Hasta la actualidad las teorías de liderazgo, así como la producción empírica ligada a éstas, han dedicado gran parte de sus esfuerzos a la explicación de una gama completa de los estilos de liderazgo. En la década de los años treinta se realizó una de las primeras investigaciones por parte de la Universidad de Lowa acerca los tipos 
de liderazgo, a cargo de Kurt Lewin y un grupo de colaboradores, quienes identificaron tres tipos básicos de estilos de liderazgo: Liderazgo autocrático (autoritario), Liderazgo liberal o de rienda suelta (Laissez Faire), y, Liderazgo participativo o democrático. Sin embargo la gama de liderazgos ha ido desarrollándose a lo largo del tiempo, ampliando la tipología de estilos concretos (Bass, 1985), y convirtiéndose en el foco central en las teorías de liderazgo (Van Engen \& Willemsen, 2000), sobre todo en las últimas dos décadas. Estas teorías suponen en términos generales que una persona que ejerce el rol de líder es percibida de una manera transformadora cuando inspira en los subordinados altos niveles de esfuerzo y dedicación, que es percibida de un modo transaccional al reconocer responsabilidades de los subordinados, premiando el cumplimiento de los objetivos y la corrección de las metas, y que un líder se considera en modo laissez-faire cuando su actividad está presidida por una evitación pasiva (Bass \& Avolio, 1993).

Entre estos tres estilos de liderazgo, el liderazgo transformacional ha resultado de especial interés para el estudio y análisis, por el volumen de literatura investigadora que ha producido. Su principal precursor ha sido Bass (1985), quien describe a este tipo de liderazgo a partir de los efectos extraordinarios que produce el líder en los miembros de una organización a través de su influencia personal y de la concientización acerca de la importancia y el valor que revisten los resultados obtenidos tras realizar las tareas asignadas. Nader y Castro Solano (2007) sostienen que el modelo explicativo de Bass hunde sus raíces en las ideas originales de House (1971) y Burns (1978) sobre el liderazgo carismático y transformacional, respectivamente. Con respecto a las características que presentan los líderes transformacionales, Bass y Riggio (2006) plantean que la gama de conductas que pueden mostrar éstos es amplia. A nivel conceptual, los autores afirman que los líderes transformacionales se proponen como ejemplos a seguir (carisma o influencia idealizada), proveen significado a las acciones de sus subordinados (inspiración), alientan la búsqueda de soluciones alternativas a problemas cotidianos (estimulación intelectual) y suelen preocuparse por las necesidades individuales de sus subordinados (consideración individualizada) (Nader \& Castro Solano, 2007). Todos estos elementos propician el registro de dimensionalidades internas en los estilos de liderazgo, que se hallan sintetizadas en la Cuadro 1.

En términos no ya teóricos, sino de evidencias empíricas, el liderazgo transformacional también ha sido objeto de una creciente atención investigadora por el impacto que ha mostrado tener en diversos ámbitos de la gestión organizacional. Dumdum, Lowe y Avolio (2002) Ilevaron a cabo meta-análisis de estudios en torno al liderazgo transformacional y transaccional, y su correlación con la efectividad y satisfacción, en donde se señala que las investigaciones previas generan resultados como conocimientos, experiencia y 
rendimiento a través de cambios en diversos aspectos afectivos, conductuales, cognitivos y de desempeño organizacional. Howell y Avolio (1993), por su parte, encontraron que el liderazgo transformacional en gerentes predijo un mayor rendimiento en los negocios consolidados luego de un año.

Dado el carácter procesual y adquirido de las actitudes y conductas propias del liderazgo transformacional, diversas investigaciones han depositado especial énfasis en el análisis de impacto de los procesos de capacitación en liderazgo transformacional. En este sentido, investigaciones experimentales como la de Barling, Weber y Kelloway (1996) han indicado la importancia del entrenamiento de liderazgo, y proporcionan evidencia de que el liderazgo transformacional puede provocar cambios en la percepción de los miembros de la organización, lo cual en estudios como el de Dvir, Eden, Avolio y Shamir (2002) se traduce en que los subordinados señalaron altas calificaciones en los líderes que han tenido entrenamiento en conductas transformacionales. Dichos resultados, que recogen el impacto del cambio comportamental a través de procesos de capacitación, coinciden con otras investigaciones (Avolio, Walumbwa \& Weber, 2009), donde se identifican al menos cuatro áreas de comportamiento en los que afecta una intervención o entrenamiento sobre liderazgo: el área afectiva, el rendimiento cognitivo, el área conductual y área organizacional.

Debido a este nexo entre procesos de entrenamiento y desarrollo de habilidades transformacionales de liderazgo, se postula la idea del interés investigador en relación a los cambios comportamentales que acompañan a las acciones de capacitación y el grado de impacto percibido por los líderes. Pero cabe preguntarse si estos cambios se producen en la misma magnitud en función de las características sociodemográficas y socio-laborales propias de los líderes. Esta cuestión precisamente es la que centra la atención del presente texto.

Cuadro 1. Estilos de liderazgo y dimensiones internas

\begin{tabular}{|l|l|l|}
\hline \multicolumn{3}{|c|}{ ESTILOS DE LIDERAZGO } \\
\hline \multicolumn{1}{|c|}{$\begin{array}{l}\text { LIDERAZGO } \\
\text { TRANSFORMACIONAL }\end{array}$} & LIDERAZGO TRANSACCIONAL & $\begin{array}{l}\text { LIDERAZGO LAISSEZ-FAIRE } \\
\text { (PASIVO) }\end{array}$ \\
\hline Definición & Definición & Definición \\
\hline $\begin{array}{l}\text { El líder, articula una visión e inspira a } \\
\text { sus seguidores, posee capacidad de } \\
\text { motivar y crea un ambiente favorable } \\
\text { para el cambio organizacional }\end{array}$ & $\begin{array}{l}\text { El líder, identifica que es lo que } \\
\text { necesitan sus subordinados } \\
\text { para cumplir sus objetios, } \\
\text { aclaran funciones y tareas } \\
\text { organizacionales permiten el el quiere servir a } \\
\text { desempeño y toman en cuenta } \\
\text { otras personas. Reconoce } \\
\text { su responsabilidad moral } \\
\text { no ante el éxito de la } \\
\text { organización sino además } \\
\text { lasia sus subordinados, hacia } \\
\text { seguidoridades sociales de sus }\end{array}$ \\
\hline
\end{tabular}




\begin{tabular}{|c|c|c|}
\hline Dimensiones & Dimensiones & Dimensiones \\
\hline $\begin{array}{l}\text { 1.-Carisma o Influencia Idealizada } \\
\text { Proporciona una visión y un sentido } \\
\text { de misión, infunde orgullo, merece } \\
\text { respeto y confianza. } \\
\text { 2.- Motivación Inspiracional } \\
\text { Capacidad del líder de comunicar su } \\
\text { visión. Utiliza símbolos para enfocar } \\
\text { los esfuerzos, expresa propósitos } \\
\text { importantes empleando medios } \\
\text { simples } \\
\text { 3.-Estimulación intelectual } \\
\text { Capacidad del líder para hacer } \\
\text { que sus subordinados piensen } \\
\text { de manera creativa e innovadora. } \\
\text { Promueve la inteligencia, la } \\
\text { racionalidad y una cuidadosa } \\
\text { solución de problemas. } \\
\text { 4.-Consideración Individualizada } \\
\text { (Atributos) } \\
\text { Capacidad del líder para prestar } \\
\text { atención personal a todos los } \\
\text { miembros de su equipo. } \\
\text { 5.-Consideración Individualizada } \\
\text { (Conducta) } \\
\text { Brinda atención personal, trata } \\
\text { a cada persona individualmente, } \\
\text { asesora, impulsa. }\end{array}$ & $\begin{array}{l}\text { 1. Premio o Recompensa } \\
\text { contingente . } \\
\text { Intercambio de premios } \\
\text { por esfuerzos o promesa } \\
\text { de premios por un buen } \\
\text { desempeño, reconocimiento } \\
\text { del éxito. } \\
\text { 2. Administración por excepción } \\
\text { ( activo) } \\
\text { Busca las desviaciones de las } \\
\text { reglas y de los patrones para } \\
\text { así aplicar acciones correctivas. } \\
\text { 3. Administración por excepción } \\
\text { (pasivo) } \\
\text { El líder interviene } \\
\text { exclusivamente cuando no } \\
\text { se alcanzan los parametros, } \\
\text { cuando hay que hacer } \\
\text { correccciones o cambios en las } \\
\text { conductas de los seguidores }\end{array}$ & $\begin{array}{l}\text { 1.Laissez-Faire } \\
\text { Ausencia de Transacciones, } \\
\text { Renuncia a las } \\
\text { responsabilidadades, evita } \\
\text { tomar decisiones, usa su } \\
\text { autoridad }\end{array}$ \\
\hline
\end{tabular}

Fuente: Elaboración propia a partir de "From Transactional to Transformational Leadership: Leaming to Share y the visión”, Organizational Dynamics, 1990, p 22 y, L Manuel Guillen Parra en su libro “ la ética en las organizaciones”Ed. Pearson, 2006 


\section{Estado de la cuestión e hipótesis de trabajo: Factores explicativos en el desarrollo de estilos de liderazgo}

En las últimas décadas se ha registrado un aumento de investigaciones que centran su interés en la influencia de diferentes variables sociodemográficas sobre los espacios, estructuras de modelos y estilos diferentes de liderazgo. Derivado de este interés creciente, la investigación sobre el estilo de liderazgo de hombres y mujeres y el análisis de sus diferencias de género ha ganado atención (Eagly \& Carli, 2003; Cuadrado, 2002 \& 2003). Avolio et al. (2009), señalan la importancia de analizar los efectos de género, tanto en los líderes como en sus seguidores. En este sentido, algunas investigaciones realizadas determinan que las mujeres tienen un estilo de liderazgo más transformacional que los hombres (por ejemplo Carless, Wearin \& Mann, 2000; Eagly \& Johannesen-Schmidt, 2001; Eagly, Johannesen-Schmidt \& Van Engen, 2003; Pérez \& Camps del Valle, 2011), 0 simplemente que el estilo de liderazgo de los hombres se caracteriza por ser más direccional con una conducta de recompensa contingente, es decir un liderazgo más transaccional (Bass, 1998; Bass \& Avolio, 1994, Bass, Avolio \& Atwater, 1996; Eagly \& JohannesenSchmidt, 2001; Druskat, 1994; Sarrió, Barberá, Ramos \& Candela, 2002; Guirado, Alonso \& Navas, 2003).

Estas posibles diferencias en el comportamiento entre hombres y mujeres podría explicarse a través de la teoría de los roles sociales (Eagly, Wood \& Diekman, 2000), según la cual las diferencias y similitudes de sexo generan expectativas en las personas en función de su género como hombre o mujer, a partir de los roles de género desplegados. En la investigación llevada a cabo por Eagly y Johnson (1990) los resultados mostraron que las mujeres lideraban con estilos más horizontales y participativos que los hombres y que éstos lo hacian de forma más autocrática o directiva que las mujeres. Druskat (1994), por su parte, revelaba que las mujeres puntúan significativamente por encima de los hombres en factores como consideración individualizada, estimulación intelectual y motivación inspiracional. En otras investigaciones las mujeres presentaban diferencias estadísticamente significativas además en la dimensión de influencia idealizada (Bass \& Avolio, 1994), así como calificaciones más altas en la consideración individualizada con respecto al liderazgo transformacional (Eagly \& Johannesen-Schmidt, 2001), y un puntaje significativo de recompensa contingente (Eagly \& Johannesen-Schmidt, 2001; Eagly, Johannesen-Schmidt \& Van Engen, 2003). Según Cuadrado (2002, 2003), las mujeres lideresas se describían a sí mismas aludiendo a una serie de características propias del liderazgo transformacional, mientras que los líderes hombres describían su estilo de liderazgo en términos de transacciones o intercambios con sus subordinados. 
También Loden (1985) mantiene que frente al estilo de dirección masculina, caracterizada por la competitividad, la autoridad jerárquica, un alto control del líder y la resolución analítica de problemas, las mujeres prefieren y tienden a comportarse siguiendo un estilo de liderazgo alternativo, que ella denomina femenino. Este estilo está caracterizado por la cooperación, la colaboración entre el líder y los subordinados y la resolución de problemas basada en la intuición, la empatía y la racionalidad (Cuadrado, 2002, 2003). Una de las tesis defendidas en la literatura por lo tanto sostiene que las mujeres tienen un estilo de liderazgo propio y diferente al de los hombres.

Pero no todos los resultados investigadores indican el mismo tipo de resultados. Algunas investigaciones organizacionales no muestran diferencias significativas para hombres y mujeres en los estilos de liderazgo (por ejemplo Engen, Leeden \& Willemsen; 2001; Cuadrado \& Molero, 2002), incluso en el caso de estudios en los que los participantes no ocupaban roles de liderazgo (Guirado, Alonso \& Navas, 2003).

Respecto al análisis de la variable edad la literatura investigadora también muestra resultados ambiguos. Algunas investigaciones señalan que no se encuentran diferencias estadísticamente significativas entre el estilo de liderazgo y la edad (Pérez \& Camps del Valle, 2011). Perez y Camps del Valle (2011) no encontraron tampoco diferencias empíricas apreciables entre los grupos considerando las variables edad del supervisor, años de experiencia laboral, tiempo que lleva supervisando empleados, cantidad de personas que supervisa y capacitaciones realizadas sobre el tema de liderazgo. Ramírez y Sgambatti (2008), en cambio, señalan que "los estilos transformacional y transaccional totales aumentaron con la edad del encuestado, es decir, a mayor edad, mayores valores de estos estilos de liderazgo, aunque esta diferencia no fue estadísticamente significativa. Estos resultados están en línea con lo reportado por Eagly, Johannesen y Engen (2003) quienes a pesar de no haber encontrado diferencias, observaron que los encuestados más jóvenes eran menos transformacionales que aquellos más adultos". Por su parte Oshagbemi (2004) encontró que las personas jóvenes con cargos de gerencia tenían perfiles de liderazgo diferentes a los gerentes mayores, específicamente para el estilo influencia idealizada.

Otra variable, la educación, ha sido también objeto de los análisis de estilos de liderazgo, por su influencia en el proceso de construcción de saberes, desarrollo personal y habilidades profesionales que conforman las prácticas de liderazgo, lo cual en teoría contribuye a capacitar a los líderes para la innovación, cambio permanente y aprovechamiento del talento humano de su institución (Pestana, Tortoza, Díaz \& Rodríguez, 2011). Así, el grado de preparación se ha mostrado como un pilar fundamental que diferencia el grado y el modo de responsabilidad para ejecución de las tareas de liderazgo (Nader \& Castro Solano, 2007). 
En relación a la influencia de los perfiles socio-laborales en relación el estilo de liderazgo, Ramírez y Sgambatti (2008) indican que quienes trabajan para empresas públicas tienden a exhibir comportamientos más transformacionales que aquellos que trabajan en empresas privadas. Pero más allá de esta cuestión, se evidencia cierto vacío investigador en torno a la capacidad explicativa diferencial que las distintas variables ligadas al puesto de trabajo (condiciones laborales, experiencia, categoría profesional, etc.) tienen sobre el desarrollo de los distintos estilos de liderazgo.

En el análisis sobre los estilos de liderazgo no solo cabe estudiar sus componentes internos y los perfiles ligados a ellos, sino también los resultados que producen o emergen en el ejercicio de cada estilo de liderazgo. Y a este respecto la literatura investigadora coincide en que al menos hay tres tipos de resultados a considerar: el extra esfuerzo, la satisfacción y la eficacia. Estos tres elementos también deben ser incorporados como variables dentro de cualquier análisis a realizar.

Aquí, la mayoría de autores coinciden en que cuando aumenta la satisfacción laboral se experimenta mayor satisfacción con la vida personal. Dicho criterio que se plantea como un indicador de un liderazgo transformacional eficaz, que influye directamente en la felicidad laboral y por ende en la consecución de resultados positivos de los miembros de una organización (Omar, 2011). Por su parte, Bass (1999) confirma que el estilo de liderazgo transformacional, sobre todo en su factor carisma, correlaciona de manera positiva con la eficacia y la satisfacción en los equipos de trabajo, y que el factor recompensa contingente del estilo de liderazgo transaccional, aunque en menor medida, también correlaciona de forma positiva con los resultados de eficacia y satisfacción. De hecho, La investigación realizada por Omar indicó que cuando los miembros de una organización visualizan que la persona que ejerce como líder aprecia sus contribuciones, motiva su proceder y atiende sus necesidades, se incrementa la satisfacción laboral. Estos resultados se refuerzan cuando los atributos que más influyen sobre la satisfacción laboral de los miembros son la percepción del supervisor como un líder carismático (influencia idealizada), y que toma decisiones basadas fundamentalmente en la razón y no en la emoción (estimulación intelectual), todos ellos componentes propios de las tesis del liderazgo transformacional. En los mismos términos Yang (2009) coincide al afirmar que el estilo de liderazgo transformador contribuye a la satisfacción laboral al generar motivación inspiradora y estímulo intelectual.

Por todo ello entendemos de interés contribuir con hallazgos empíricos al debate investigador y académico que aborda la relación entre variables propias del perfil de los sujetos con responsabilidades de liderazgo y las características del estilo de liderazgo desarrollado por éstos, así como los resultados que se derivan de este ejercicio. Las variables definitorias del perfil sociodemográfico y sociolaboral de los sujetos, planteadas en los aparados 
introductorios, constituyen pues el sustrato analítico a partir del cual el presente texto se plantea los objetivos e hipótesis de trabajo. $Y$ estas preguntas investigadoras se articulan en torno a dos objetivos básicos. En primer averiguar si el desarrollo de un estilo de liderazgo concreto en los sujetos responde en mayor medida a cuestiones de carácter descriptivo (edad, sexo, o etnia) o por el contrario a variables de carácter adquirido en su experiencia (posición laboral, sector de empleo, dedicación laboral o años de dedicación). Y como segundo objetivo investigador, se plantea analizar si las dimensiones propias del liderazgo transformacional poseen especial predominio sobre la dimensiones de los otros estilos de liderazgo para la explicación del comportamiento de variables identificadas como resultados en el ejercicio del liderazgo.

\section{Metodología.}

Para analizar la incidencia del proceso de formación en liderazgo transformacional. E grupo de análisis objeto de estudio de la presente instigación lo comprenden un grupo diverso de líderes sociales provenientes del sector público, privado y mixto de las ciudades de Cuenca, Guayaquil, Portoviejo, Riobamba y Tena, que se matricularon en el "Programa de capacitación en fortalecimiento socio-organizacional para un liderazgo transformacional con la generación de conocimientos y análisis de propuestas que dinamicen el desarrollo territorial en el Ecuador, 2014".

El Programa de capacitación se ejecutó en el período académico septiembre del 2014 a marzo del 2015, con una duración de 7 meses en la que se impartieron 11 módulos. Cada módulo contaba con 16 horas presenciales y virtuales, y 39 horas estimadas para la elaboración de proyectos, tarea, lectura y otros. Los 11 módulos responden a tres ejes fundamentales de formación de un líder transformador: 1) Técnicos (relacionado a temas de desarrollo), 2) Nuevas tecnologías aplicadas al desarrollo; 3) Instrumental de apoyo social.

De las cinco sedes en las que se abrió el programa de formación, participaron un total de 165 líderes que registraron asistencia regular a la capacitación. Dada la gran heterogeneidad de la población de estudio, debido a diversas características socio-demográficas, sociolaborales y socio-económicas, se planteó trabajar con una muestra representativa en base a un diseño muestral que contempló un muestreo aleatorio estratificado con afijación simple, con la disposición de estratos homogéneos por sexo y grupos de edad, asumiendo un nivel de confianza del $95,5 \%$, un margen de error máximo permisible del $5 \%$ y probabilidad estandarizada de éxito $p=50 \%$ y de fracaso $q=50 \%$. A partir de los cual, en la presente investigación se trabajó con una muestra representativa de 116 participantes. 
Para levantar información primaria de los participantes sobre el impacto del programa de formación se decidió utilizar la técnica por encuesta a través de la metodología cuantitativa. Para ello se realizó una revisión minuciosa del estado de arte y los instrumentos utilizados de otras investigaciones sobre información concerniente a los estilos de liderazgo de rango completo y sus resultados. Encontrándose que en la comunidad científica, como instrumento ampliamente validado en esta temática está el Cuestionario Multifactorial de Liderazgo (MLQ).

EI MLQ mide la autopercepción de la conducta de los líderes, con 45 ítems de escala Likert abarca 9 dimensiones del liderazgo, que conforman los tres estilos de liderazgo de acuerdo a la teoría de rango total que propone Bass (1985): Liderazgo transformacional, transaccional y Laissez-Faire; así como también, mide los tres resultados esperados del Liderazgo: Extra esfuerzo, eficacia y satisfacción. Por parte del equipo investigador se vio la necesidad de agregar al cuestionario original variables de escalas nominales y ordinales, con categorías dicotómicas y politómicas, para medir los perfiles socio-demográficos y socio-laborales de los líderes. Las variables socio-demográficas incorporadas fueron: edad, sexo, lugar de trabajo/estudios actuales, lugar de residencia cuando tenía 16 años y nivel educativo. Y las variables socio-laborales incorporadas fueron: sector donde trabaja (público, privado, social), experiencia laboral, jornada laboral y ocupación en el trabajo.

Con la finalidad de realizar un estudio longitudinal del impacto del entrenamiento del programa de formación, se plantea tres tiempos de aplicación del cuestionario del MLQ, en la mitad, al final y después del proceso de capacitación. Para la publicación del presente artículo se toman los primeros resultados obtenidos en: Fase I: Primera ronda de encuestas (R1) que se aplicó después del módulo 4, "Competitividad, emprendimiento e innovación tecnológica", en la ejecución del primer módulo de refuerzo de los tres módulos anteriores.

Los resultados fueron generados con el programa estadístico SPSS (Statistical Package for the Social Sciences) versión 20, utilizando las técnicas: ANOVA de un factor para explicar los factores socio-demográficas y socio-laborales que inciden en diferencias significativas en los estilos de liderazgo; Así como, también se utilizan técnicas de correlación y regresión múltiple, con las cuales se probaron las relaciones y la influencia explicativa de los estilos de liderazgo de rango completo en los resultados esperados del liderazgo.

\section{Análisis y resultados.}

El primer objetivo analítico, como hemos comentado, centra su interés en la identificación de las variables contextuales que poseen una mayor carga explicativa en el desarrollo de los distintos estilos de liderazgo. En este sentido cabe plantearse como hipótesis de trabajo si las variables relativas al contexto socio-laboral, a partir de la atribución de un carácter 
procesual al desarrollo de los estilos de liderazgo, posee una mayor incidencia sobre éstos en relación a las variables sociodemográficas, las cuales se definen por un componente netamente descriptivo 0 anterior a la experiencia laboral del sujeto. Para ello se han asumido en el análisis como variables vinculadas a las características sociodemográficas el sexo, la edad, la etnia y el nivel educativo. Por su parte, para el ámbito socio-laboral se han establecido como variables de segmentación el sector de empleo, la dedicación laboral y los años de experiencia en el empleo.

Dentro del marco analítico de este primer objetivo, en la tabla 1 se puede observar, a partir de un análisis ANOVA de un factor, cómo los resultados indican que son las variables sociodemográficas las que poseen una mayor significación empírica en el valor que presenta el estadístico F por su capacidad explicativa sobre el desarrollo de los distintos estilos de liderazgo de las personas, (el sexo influye sobre el liderazgo transaccional, la edad y el nivel de estudios sobre el liderazgo transformacional y transaccional, y la etnia sobre el liderazgo pasivo). Las variables socio-laborales, es decir, las características del puesto de trabajo y el perfil profesional de los sujetos, por el contrario no presentan una capacidad explicativa empíricamente significativa sobre el estilo de liderazgo que despliegan las personas.

Los resultados refutan la hipótesis de partida pero son a nuestro juicio muy interesantes, puesto que marcan una explicación en cierto modo determinista sobre el estilo de liderazgo que desarrollan los sujetos. Son las variables ajenas al ámbito laboral las que definen la forma en que las personas desarrollan su estilo de liderazgo, mientras que variables contextuales en torno a la situación laboral en cuyo ámbito el sujeto lleva a cabo las acciones de liderazgo, y que a priori podría esperarse que ejercieran influencia sobre los estilos, no lo hacen.

Tabla 1. Perfil socio-demográfico y socio-laboral en los estilos de liderazgo

\begin{tabular}{lccc}
\hline SEXO & $\begin{array}{c}\text { Liderazgo } \\
\text { transformacional }\end{array}$ & $\begin{array}{c}\text { Liderazgo } \\
\text { transaccional }\end{array}$ & $\begin{array}{c}\text { Liderazgo } \\
\text { Pasivo }\end{array}$ \\
\cline { 2 - 4 } Hombre & 4.34 & 4.13 & 2.68 \\
Mujer & 4.24 & 3.82 & 2.55 \\
F de ANOVA & 1,623 & 10,121 & 1,324 \\
Valor p & 0,205 & $\mathbf{0 , 0 0 2}$ & 0,252 \\
\hline
\end{tabular}




\begin{tabular}{|c|c|c|c|}
\hline \multicolumn{4}{|l|}{ EDAD } \\
\hline Hasta 28 años & 4.09 & 3.78 & 2.63 \\
\hline $29-35$ & 4.25 & 3.87 & 2.50 \\
\hline $36-44$ & 4.41 & 4.13 & 2.59 \\
\hline 45 y más & 4.40 & 4.14 & 2.77 \\
\hline F de ANOVA & 4,132 & 3,474 & 0,864 \\
\hline Valor $p$ & 0,008 & 0,018 & 0,462 \\
\hline \multicolumn{4}{|l|}{ NIVEL EDUCATIVO } \\
\hline Primer y segundo nivel & 3.98 & 3.80 & 2.96 \\
\hline Tercer Nivel & 4.25 & 3.85 & 2.55 \\
\hline Cuarto nivel & 4.45 & 4.21 & 2.59 \\
\hline F de ANOVA & 7,589 & 6,817 & 2,118 \\
\hline Valor $p$ & 0,001 & 0,002 & 0,125 \\
\hline \multicolumn{4}{|l|}{ ETNIA } \\
\hline Indígena & 4.19 & 3.91 & 3.07 \\
\hline Blanco & 4.36 & 4.08 & 2.37 \\
\hline Mestizo & 4.28 & 3.97 & 2.55 \\
\hline Otras & 4.56 & 4.08 & 2.12 \\
\hline F de ANOVA & 0,734 & 0,145 & 4,426 \\
\hline Valor $p$ & 0,534 & 0,933 & 0,006 \\
\hline \multicolumn{4}{|l|}{ SECTOR DE EMPLEO } \\
\hline Sector público & 4.32 & 4.05 & 2.62 \\
\hline Sector privado & 4.40 & 3.95 & 2.47 \\
\hline Sector comunitario & 4.38 & 4.17 & 2.76 \\
\hline F de ANOVA & 0,584 & 0,598 & 0,885 \\
\hline Valor $p$ & 0,560 & 0,552 & 0,416 \\
\hline
\end{tabular}




\begin{tabular}{lccc}
\hline DEDICACIÓN LABORAL & & & \\
A tiempo completo & 4.36 & 4.08 & 2.58 \\
A tiempo parcial & 4.31 & 3.86 & 2.69 \\
\hline F de ANOVA & 0,336 & 2,676 & 0,441 \\
Valor p & 0,563 & 0,105 & 0,508 \\
\hline AÑOS DE SERVICIO DE & & & \\
EMPLEO & 4.23 & 3.80 & 2.46 \\
Menos de 1 año & 4.36 & 4.06 & 2.62 \\
Entre 1 y 5 años & 4.37 & 4.17 & 2.64 \\
Entre 6 y 10 años & 4.48 & 4.16 & 2.72 \\
Más de 10 años & 1,411 & 2,146 & 0,517 \\
F de ANOVA & 0,245 & 0,100 & 0,672 \\
Valor $p$ & & & \\
\hline
\end{tabular}

En negrita diferencias significativas para valor $p<0,05$ $n=116$

Fuente: Cuestionario MLQ adaptado al contexto ecuatoriano en el marco del proyecto de investigación "Impacto del entrenamiento en liderazgo transformacional en el comportamiento de líderes: un estudio cuasi-experimental en Ecuador".

Si entramos a valorar los resultados no ya en el análisis de los estilos de liderazgo sino en un mayor detalle haciendo uso de las dimensiones específicas internas que los definen (Tabla 2), en términos generales los resultados muestran que las variables de edad y nivel educativo ganan peso explicativo puesto que influyen sobre prácticamente todas las dimensiones. Particularmente hallamos que:

- La dimensión de consideración Individualizada está explicada por la edad (donde la personas mayores de 45 años puntúan más alto que el resto), el nivel educativo (más los de cuarto nivel), y los años en el empleo (los que llevan más de 10 años en el puesto de trabajo).

- La dimensión de estimulación intelectual presenta diferencias significativas en las variables sexo (los hombres puntúan más alto), la edad (puntúan más alto las personas mayores de 45 años), y el nivel educativo (puntúan más alto las personas con cuarto nivel). 
- La motivación inspiracional queda explicada únicamente por la edad (puntuaciones más altas en las personas entre 36 y 44 años).

- La influencia idealizada (atributos) está explicada por el nivel educativo (mayores puntuaciones registradas en el cuarto nivel) y el sector de empleo (personas que desarrollan su empleo en el sector privado).

- La influencia idealizada (comportamiento) no posee ninguna variable diferencial explicativa.

- La dirección por excepción activa por su parte queda explicada por el sexo (puntuaciones más altas en los hombres), el nivel educativo (cuarto nivel) y el tipo de empleo (puntúan más aquellas personas que se encuentran empleadas a tiempo completo).

- La dimensión de recompensa contingente se presenta explicada por la edad (puntuaciones más altas las personas con más de 45 años) y el nivel educativo (personas con cuarto nivel de educación).

- La dirección por excepción pasiva está explicada únicamente por la etnia (puntuaciones más altas en la población indígena).

- $\quad Y$ finalmente la dimensión de laissez-faire queda explicada en términos significativos por el nivel educativo (mayores puntuaciones en personas con primer y segundo nivel) y la etnia (puntuaciones superiores en la población indígena).

Tabla 2. Perfil socio-demográfico y socio-laboral, en las dimensiones internas de estilos de liderazgo

\begin{tabular}{|c|c|c|c|c|c|c|c|c|c|}
\hline & \multicolumn{5}{|c|}{$\begin{array}{c}\text { Liderazgo } \\
\text { transformacional }\end{array}$} & \multicolumn{2}{|c|}{$\begin{array}{l}\text { Liderazgo } \\
\text { transaccional }\end{array}$} & \multicolumn{2}{|c|}{$\begin{array}{l}\text { Liderazgo } \\
\text { pasivo }\end{array}$} \\
\hline & 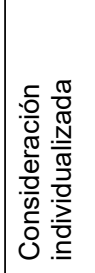 & 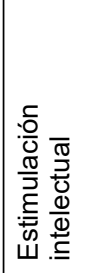 & 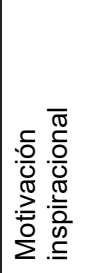 & 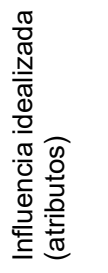 & 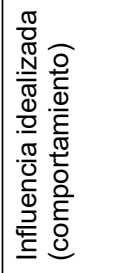 & 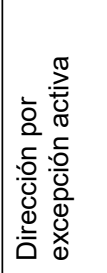 & 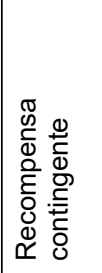 & 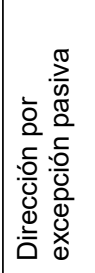 & 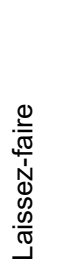 \\
\hline $\begin{array}{l}\text { SEXO } \\
\text { Hombre } \\
\text { Mujer }\end{array}$ & $\begin{array}{l}4.40 \\
4.32\end{array}$ & $\begin{array}{l}4.19 \\
3.97\end{array}$ & $\begin{array}{l}4.51 \\
4.52\end{array}$ & $\begin{array}{l}4.31 \\
4.18\end{array}$ & $\begin{array}{l}4.26 \\
4.18\end{array}$ & $\begin{array}{l}3.79 \\
3.29\end{array}$ & $\begin{array}{l}4.47 \\
4.35\end{array}$ & $\begin{array}{l}2.69 \\
2.50\end{array}$ & $\begin{array}{l}2.67 \\
2.60\end{array}$ \\
\hline $\mathrm{F}$ de ANOVA & 0,501 & 4,467 & 0,008 & 1,662 & 0,805 & 13,459 & 1,469 & 1,667 & 0,355 \\
\hline Valor $p$ & 0,481 & 0,037 & 0,928 & 0,200 & 0,372 & 0,000 & 0,228 & 0,199 & 0,552 \\
\hline
\end{tabular}




\begin{tabular}{|c|c|c|c|c|c|c|c|c|c|}
\hline EDAD & & & & & & & & & \\
\hline Hasta 28 años & 4.13 & 3.85 & 4.33 & 4.06 & 4.06 & 3.31 & 4.25 & 2.68 & 2.57 \\
\hline $29-35$ & 4.31 & 4.03 & 4.48 & 4.23 & 4.18 & 3.47 & 4.27 & 2.46 & 2.53 \\
\hline $36-44$ & 4.50 & 4.21 & 4.68 & 4.35 & 4.33 & 3.75 & 4.51 & 2.46 & 2.72 \\
\hline 45 y más & 4.52 & 4.26 & 4.57 & 4.35 & 4.32 & 3.63 & 4.65 & 2.82 & 2.73 \\
\hline $\mathrm{F}$ de ANOVA & 2,760 & 3,083 & 2,679 & 1,961 & 1,950 & 1,866 & 4,141 & 1,232 & 0,679 \\
\hline Valor $p$ & 0,046 & 0,032 & 0,049 & 0,124 & 0,126 & 0,139 & 0,008 & 0,301 & 0,566 \\
\hline NIVEL & & & & & & & & & \\
\hline EDUCATIVO & 3.89 & 3.66 & 4.31 & 4.00 & 4.04 & 3.52 & 4.08 & 2.85 & 3.08 \\
\hline Primer y segundo & 4.34 & 4.00 & 4.51 & 4.19 & 4.19 & 3.34 & 4.36 & 2.59 & 2.52 \\
\hline nivel & 4.55 & 4.33 & 4.60 & 4.40 & 4.35 & 3.82 & 4.59 & 2.50 & 2.68 \\
\hline Tercer Nivel & & & & & & & & & \\
\hline Cuarto nivel & & & & & & & & & \\
\hline F de ANOVA & 6,984 & 8,866 & 1,600 & 3,403 & 2,648 & 5,176 & 5,845 & 0,835 & 3,914 \\
\hline Valor $p$ & 0,001 & 0,000 & 0,207 & 0,037 & 0,075 & 0,007 & 0,004 & 0,436 & 0,007 \\
\hline ETNIA & & & & & & & & & \\
\hline Indígena & 4.25 & 3.94 & 4.62 & 4.19 & 3.97 & 3.56 & 4.26 & 3.12 & 3.02 \\
\hline Blanc & 4.41 & 4.25 & 4.25 & 4.50 & 4.41 & 3.91 & 4.25 & 2.41 & 2.33 \\
\hline Mest & 4.36 & 4.08 & 4.49 & 4.2 & 4.25 & 3.51 & 4.43 & 2.53 & 2.58 \\
\hline Otras & 4.75 & 4.33 & 4.83 & 4.58 & 4.33 & 3.66 & 4.50 & 2.00 & 2.25 \\
\hline $\mathrm{F}$ de ANOVA & 0,650 & 0,608 & 0,988 & 0,666 & 1,959 & 0,310 & 0,674 & 3,378 & 2,913 \\
\hline Valor $p$ & 0,585 & 0,611 & 0,401 & 0,575 & 0,124 & 0,818 & 0,570 & 0,021 & 0,038 \\
\hline SECTOR DE & & & & & & & & & \\
\hline EMPLEC & 4.41 & 4.14 & 4.53 & 4.20 & 4.30 & 3.68 & 4.43 & 2.55 & 2.69 \\
\hline Sector público & 450 & 4.13 & 4.64 & 4.4 & 4.27 & 3.45 & 4.45 & 2.46 & 2.48 \\
\hline Sector privado & 4.53 & 4.21 & 4.59 & 4.25 & 4.31 & 3.81 & 4.53 & 2.90 & 2.62 \\
\hline Sector comunitario & & & & & & & & & \\
\hline $\mathrm{F}$ de ANOVA & 0,408 & 0,082 & 0,599 & 3,155 & 0,037 & 1,061 & 0,136 & 0,875 & 0,996 \\
\hline Valor $p$ & 0,666 & 0,921 & 0,552 & 0,047 & 0,964 & 0,350 & 0,873 & 0,420 & 0,373 \\
\hline TIPO DE EMPLEO & & & & & & & & & \\
\hline A tiempo com & 4.45 & 4.16 & 4.58 & 4.30 & 4.31 & 3.70 & 4.46 & 2.55 & 2.61 \\
\hline A tiempo parci & 4.41 & 4.08 & 4.56 & 4.27 & 4.21 & 3.31 & 4.41 & 2.65 & 2.73 \\
\hline $\mathrm{F}$ de ANOVA & 0,092 & 0,332 & 0,035 & 0,049 & 0,774 & 4,092 & 0,34 & 0,190 & 0,556 \\
\hline Valor $p$ & 0,762 & 0,566 & 0,852 & 0,826 & 0,381 & 0,046 & 0,716 & 0,664 & 0,458 \\
\hline AÑOS EN EL & & & & & & & & & \\
\hline EMPLEO & 4.21 & 4.06 & 4.50 & 4.17 & 4.22 & 3.30 & 4.30 & 2.43 & 2.50 \\
\hline Menos de 1 año & 4.48 & 4.15 & 4.58 & 4.29 & 4.29 & 3.64 & 4.48 & 2.60 & 2.65 \\
\hline Entre 1 y 5 años & 4.40 & 4.26 & 4.51 & 4.35 & 4.32 & 3.93 & 4.40 & 2.56 & 2.73 \\
\hline $\begin{array}{l}\text { Entre } 6 \text { y } 10 \text { años } \\
\text { Más de } 10 \text { años }\end{array}$ & 4.73 & 4.12 & 4.76 & 4.44 & 4.35 & 3.69 & 4.62 & 2.75 & 2.69 \\
\hline$F$ de ANOVA & 84 & 468 & 1222 & 933 & 0257 & 2 & 4 & 0.415 & 0,460 \\
\hline Valor $\mathrm{p}$ & 0.031 & 0705 & 0306 & 0428 & 0856 & 0083 & 85 & 0713 & 711 \\
\hline valor $p$ & 0,031 & 0,105 & 0,306 & 0,428 & 0,050 & 0,003 & 0,200 & 0,143 & $0, \pi \mathrm{I}$ \\
\hline
\end{tabular}

En negrita diferencias significativas para valor $p<0,05$

$\mathrm{n}=116$

Fuente: Cuestionario MLQ adaptado al contexto ecuatoriano en el marco del proyecto de investigación

"Impacto del entrenamiento en liderazgo transformacional en el comportamiento de líderes: un estudio cuasi-experimental en Ecuador". 
El segundo objetivo investigador propuesto a análisis recordemos que se proponía indagar acerca de la posible influencia diferencial que los distintos estilos de liderazgo tienen sobre los resultados en el ejercicio de éste. Aquí la hipótesis de trabajo se formula en términos de especial interés sobre la figura del liderazgo transformacional, que como hemos comentado constituye el estilo de liderazgo con mayor interés en la literatura investigadora. En este sentido la hipótesis formulada enuncia que las dimensiones propias del liderazgo transformacional explicarán, en mejor medida que el resto, los resultados considerados como exitosos del liderazgo, que se concretan para el análisis en las variables extra esfuerzo, satisfacción y eficiencia.

A este fin la tabla 3 presenta la capacidad predictiva de los distintos estilos de liderazgo a través de sus dimensiones internas sobre las variables resultado: eficacia, extra esfuerzo y satisfacción. Para ello se ha planteado la construcción de modelos de regresión múltiple, donde las dimensiones de los estilos de liderazgo han sido consideradas como variables independientes y las variables resultado como dependientes. Como se puede apreciar en la tabla, los tres modelos muestran una elevada correlación interna a través de la $\mathrm{R}$ de Pearson y una capacidad predictiva considerable (en los tres casos $\mathrm{R}^{2}$ presenta valores superiores a 0.5), explicando amplios porcentajes de varianza del conjunto de variables dependientes. Los resultados de los modelos de regresión indican un predominio de las dimensiones propias del liderazgo transformacional sobre el resto de dimensiones, especialmente en el caso de la consideración individualizada, que resulta constituirse como una variable sinérgica en la capacidad predictiva sobre las tres variables consideradas como resultados exitosos del ejercicio de liderazgo.

En términos detallados, para la variable de resultado "eficacia" presentan valores significativos como predictoras la consideración individualizada, la motivación inspiracional y la dirección por excepción activa. Para la variable "satisfacción" actúan como variables predictoras significativas la consideración individualizada, la influencia idealizada (atributos) y laissez-faire. Finalmente, la variable "esfuerzo extra", presenta como variables predictoras significativas las dimensiones de consideración individualizada, motivación inspiracional, dirección por excepción activa y recompensa contingente. El resultado por lo tanto, aunque con ciertas matizaciones para cada variable de resultado, confirma la hipótesis de la importancia de los atributos propios del liderazgo transformacional en la explicación del comportamiento de las variables consideradas como resultados exitosos del ejercicio del liderazgo. 
Tabla 3. Modelos de regresión múltiple para los resultados de los estilos de liderazgo

\begin{tabular}{|c|c|c|c|c|c|c|c|}
\hline \multirow[b]{2}{*}{$\begin{array}{l}\text { Estilo de } \\
\text { liderazgo }\end{array}$} & \multirow[b]{2}{*}{ Dimensiones } & \multicolumn{2}{|c|}{$\begin{array}{l}\text { Modelo de } \\
\text { regresión } \\
\text { EFICACIA }\end{array}$} & \multicolumn{2}{|c|}{$\begin{array}{l}\text { Modelo de } \\
\text { regresión } \\
\text { SATISFACCIÓN }\end{array}$} & \multicolumn{2}{|c|}{$\begin{array}{c}\text { Modelo de } \\
\text { regresión } \\
\text { ESFUERZO } \\
\text { EXTRA } \\
\text { R de Pearson = } \\
0,768 \\
\mathrm{R}^{2}=0,589\end{array}$} \\
\hline & & $\begin{array}{l}\text { Coef. B } \\
\text { Estándar. }\end{array}$ & Sig. & $\begin{array}{l}\text { Coef. B } \\
\text { Estándar. }\end{array}$ & Sig. & $\begin{array}{l}\text { Coef. B } \\
\text { Estándar. }\end{array}$ & Sig. \\
\hline \multirow[t]{5}{*}{$\begin{array}{l}\text { Liderazgo } \\
\text { transformacional }\end{array}$} & $\begin{array}{l}\text { Consideración } \\
\text { Individualizada }\end{array}$ & .326 & 0,001 & .395 & 0,000 & .282 & 0,003 \\
\hline & Estimulación intelectual & .058 & 0,657 & .140 & 0,142 & .125 & 0,159 \\
\hline & Motivación inspiracional & .179 & 0,038 & .155 & 0,092 & .276 & 0,002 \\
\hline & $\begin{array}{l}\text { Influencia idealizada: } \\
\text { atributos }\end{array}$ & .082 & 0,333 & .210 & 0,022 & -.044 & 0,607 \\
\hline & $\begin{array}{l}\text { Influencia idealizada: } \\
\text { comportamiento }\end{array}$ & .094 & 0,274 & -.092 & 0,316 & .000 & 0,999 \\
\hline \multirow[t]{2}{*}{$\begin{array}{l}\text { Liderazgo } \\
\text { transaccional }\end{array}$} & $\begin{array}{l}\text { Dirección por excepción } \\
\text { activa }\end{array}$ & .168 & 0,028 & -.028 & 0,726 & .165 & 0,030 \\
\hline & Recompensa contingente & .107 & 0,243 & .076 & 0,436 & .189 & 0,039 \\
\hline \multirow[t]{2}{*}{$\begin{array}{l}\text { Liderazgo } \\
\text { pasivo }\end{array}$} & $\begin{array}{l}\text { La dirección por excepción } \\
\text { pasiva }\end{array}$ & -.004 & 0,952 & -.003 & 0,967 & .011 & 0,878 \\
\hline & Laissez-faire & .069 & 0,369 & .205 & 0,014 & .063 & 0,407 \\
\hline
\end{tabular}

En negrita diferencias significativas para valor $p<0,05$ $n=116$

Fuente: Cuestionario MLQ adaptado al contexto ecuatoriano en el marco del proyecto de investigación "Impacto del entrenamiento en liderazgo transformacional en el comportamiento de líderes: un estudio cuasi-experimental en Ecuador". 


\section{Discusión.}

A partir de la aplicación empírica realizada, de la generación de resultados a través del análisis ANOVA de un factor y del análisis de regresión y correlación múltiple con la finalidad de confirmar empíricamente el dimensionamiento interno de los ítems que corresponden a los estilos de liderazgo, se pueden considerar algunos hallazgos destacados:

En primer lugar, los resultados obtenidos indican que existen diferencias significativas por género en el estilo de liderazgo transaccional, esto es que los hombres demuestran ejercer un liderazgo más transaccional que las mujeres. Confirmándose lo planteado (Bass, 1998; Bass y Avolio, 1994, Bass, Avolio y Atwater, 1996; Eagly y Johannesen-Schmidt, 2001; Druskat, 1994; Sarrió, Barberá, Ramos \& Candela, 2002; Guirado, I. C., Alonso, F. M., \& Navas, M., 2003) quienes manifiestan que el estilo de liderazgo de los hombres se caracteriza por ser más direccional y transaccional con una conducta de recompensa contingente. En el caso del presente estudio el estilo de liderazgo transaccional puntúa más en los hombres con una conducta dirección por excepción activa y un valor $p$ de 0,000 que indica evidencia extremadamente fuerte de que en el largo plazo se mantendría por parte de los hombres este tipo de conducta, no así en la categoría por recompensa contingente que demuestra un valor $p$ de 0.228 .

En segundo lugar, los resultados aportan con evidencias de diferencias significativas en los estilos de liderazgo transformacional y transaccional por edad. En ambos estilos de liderazgo se visualiza un liderazgo transformacional y transaccional más pronunciado en edades adultas, esto es a partir de los 36 años en adelante. La influencia de la edad adulta en el estilo de liderazgo transformacional, se evidencia de acuerdo en la tabla 3 corresponder a las categorías: consideración individualizada, estimación intelectual y motivación inspiracional. Resultados que demuestran la alta consideración y aprecio que cualquier grupo tendrá ante la trayectoria y experiencia del líder. Ratificándose con lo señalado por Ramírez \& Sgambatti (2008) "a mayor edad, mayores valores de estilo de liderazgo transformacional". Aunque esta diferencia manifestada por Ramírez \& Sgambatti (2008) no fue muy significativa, en la presente investigación aplicada al contexto ecuatoriano se demuestra que en las edades adultas se manifiesta de manera muy pronunciada un liderazgo transformacional y el hallazgo es también en el liderazgo transaccional puntuando más en este último en la categoría recompensa contingente.

En tercer lugar, el análisis de la influencia del nivel educativo en los estilos de liderazgo evidencia diferencias extremadamente significativas en el liderazgo transformacional en niveles educativos superiores con alta puntuación en la escala Likert en las categorías 
consideración individualizada, estimación intelectual e influencia idealizada. Los valores $p$ son de: 0,001 y 0,000 en las primeras dos categorías indican que en el largo plazo se estará demostrando un estilo de liderazgo transformacional en las categorías antes mencionadas cuando el nivel educativo es superior; en tanto, que en el mediano plazo se estará visualizando un estilo de liderazgo transformacional en la categoría influencia idealizada con un valor $p$ de 0,037. Así mismo, se evidencia que a mayores niveles educativos de la mano con un estilo de liderazgo transformacional se presenta el estilo de liderazgo transaccional y laissez faire que indican diferencias significativas en el largo plazo en el liderazgo transaccional con las categorías dirección por excepción activa y recompensa contingente con valores $p$ de 0,007 y 0,004 respectivamente y en el liderazgo pasivo en la categoría pasivo evitativo con un valor $p$ de 0.007 .

En cuarto lugar, el análisis de los factores organizacionales esto es nivel de influencia de variables socio-laborales en los estilos de liderazgo, permiten visualizar con un valor $p$ de 0.047 diferencias significativas en el corto plazo en el estilo de liderazgo transformacional en la categoría influencia idealizada esto es atributos del líder para desempeñarse en el ámbito público, privado o comunitario. Dato que no corrobora lo planteado por Ramírez \& Sgambatti (2008) en el sentido de que quienes trabajan para empresas públicas tienden a exhibir comportamientos más transformacionales que aquellos que trabajan para empresas privadas. No se encontraron diferencias significativas entre el sector de empleo y los estilos de liderazgo transaccional y pasivo.

Por otra parte, en cuanto a la influencia de la dedicación laboral en un estilo de liderazgo transformacional y pasivo no se encuentran diferencias significativas, más en el estilo de liderazgo transaccional se evidencia en el corto plazo con un valor $p 0.046$ diferencias en la categoría dirección por excepción activa de acuerdo al tiempo de dedicación. Así, también la experiencia laboral indica ejercer influencia en el liderazgo transformacional en la categoría consideración individualizada que demuestra una diferencia significativa en el mediano plazo con un valor $p$ de 0.031 sin encontrarse influencias significativas de esta variable es los estilos de liderazgo transaccional y pasivo. 


\section{Conclusiones.}

Finalmente, los autores (2015, p.94) decimos que:

"Los datos muestran que los estilos de liderazgo se forjan al margen e independientemente de la experiencia profesional y laboral de los sujetos. En concreto, las claves explicativas que permiten diferenciar los estilos entre unos $y$ otros individuos descansan en características que poseen una naturaleza descriptiva, o presentes en los sujetos con anterioridad al desempeño de su rol de líder. Y más concretamente las variables con capacidad explicativa estadística significativa podemos situarlas en el ámbito de los perfiles sociodemográficos (sexo, edad, nivel educativo y etnia en menor medida). En cambio, el análisis de las variables ligadas al contexto socio-laboral, en cuyo seno cabría esperar relaciones de dependencia reveladoras en el estilo de liderazgo a partir de las diferencias en el sector de empleo, la dedicación laboral o la experiencia profesional, no se encuentran diferenciaciones apreciables. Los resultados, en este sentido, ofrecen una visión relativamente determinista de los perfiles con que los sujetos desempeñan su rol de liderazgo".

A partir de ello inferimos que las dimensiones explicativas de las variables sociodemográficas ofrecen resultados mucho más convincentes y explicativos en relación a los estilos de liderazgo al explorar los atributos de los líderes que si el análisis se centra en el contexto socio-laboral y socio-profesional de éstos.

Por último, algunos autores como Lowe, Kroeck y Sivasubramaniam, (1996) citado por Guirado I., Alonso F. y Navas M., (2003, p.117) coinciden en que: "Las numerosas investigaciones realizadas con el cuestionario multifactorial MLQ han demostrado consistentemente que con el estilo de liderazgo transformacional se obtienen niveles de rendimiento y satisfacción por parte de los subordinados mucho más elevados que con el liderazgo transaccional'. Es así, que con los resultados obtenidos se verifica que en el estilo de liderazgo transaccional no se presenta diferencias significativas en la satisfacción laboral; sin embargo, en un estilo de liderazgo transformacional incrementa la satisfacción laboral en la categoría consideración individualizada y en la categoría influencia idealizada por atributos. De igual manera, ocurre con el liderazgo pasivo en la categoría pasivo evitativo. 
En lo que respecta a la variable de resultado esfuerzo extra los datos demuestran correlaciones muy significativas entre las categorías consideración individualizada y motivación inspiracional con el estilo de liderazgo transformacional y en las categorías dirección por excepción activa y recompensa contingente con el estilo de liderazgo transaccional. En la variable eficacia no incide el liderazgo pasivo, más el liderazgo transformacional y el estilo de liderazgo transaccional demuestran influenciar significativamente en las categorías consideración individualizada, motivación inspiracional y dirección por excepción respectivamente las dos primeras para el transformacional y la última en el estilo transaccional.

De esta manera, concluimos en que:

"Las dimensiones internas que integran el liderazgo transformacional, en nuestra investigación se han mostrado como buenas predictoras de los resultados esperados del liderazgo, especialmente en el caso de la consideración individualizada y la motivación inspiracional. Estas dimensiones en síntesis abordan las capacidades inter-comunicativas de las personas que ejercen el liderazgo en relación a los miembros de la organización, lo cual a partir de lo mostrado hace recomendable a nuestro juicio desarrollar sobre estos aspectos esfuerzos, tanto en el plano analítico para futuras investigaciones como en el pragmático en términos de entrenamiento de líderes, a fin de reforzar los logros del liderazgo en el ámbito organizacional". 


\section{REFERENCIAS}

Álava. G, Domínguez. L. \& Francés. F. (2015). Estilos de Liderazgo en Ecuador dentro del ámbito comunitario y laboral. Memorias del III Congreso REDU 2015, 93-96.

Avolio. B, Reichard. R, Hannah. S, Walumbwa \& Chan. A. (2009). A meta-analytic review of leadership impact: Experimental and quasi-experimental studies. The Leadership Quarterly, 20 (5), 764-784.

Avolio, B. J., Walumbwa, F. O., \& Weber, T. J. (2009). Leadership: Current theories, research, and future directions. Annual review of psychology, 60, 421-449.

Avolio, B. \& Luthans, F. (2006). The high impact leader: Moments matter for accelerating authentic leadership development.New York: McGraw-Hill.

Barling, J., Weber, T., \& Kelloway, E. K. (1996). Effects of transformational leadership training on attitudinal and financial outcomes: A field experiment. Journal of Applied Psychology, $81(6), 827$.

Bass, B. M. (1985). Leadership and performance beyond expectations. Free Press; Collier Macmillan.

Bass, B. M., \& Avolio, B. J. (1993). Leadership and organizational culture. Public administration quarterly, 112-121.

Bass. B. (1997). From transactional to transformational leadership: learning to share the visión. Organizational Dynamics, 19-31.

Bass, B. M., \& Avolio, B. J. (1994). Shatter the glass ceiling: Women may make better managers. Human resource management, 33(4), 549-560.

Bass, B. M., \& Riggio, R. E. (2006). Transformational leadership. Psychology Press.

Burns, J. M. (1978). Leadership New York.

Carless, S. A., Wearing, A. J., \& Mann, L. (2000). A short measure of transformational leadership. Journal of Business and Psychology, 14(3), 389-405.

Chiavenato, I. (2000). Comportamiento Organizacional en el trabajo. Mexico: Mc Graw Hill.

Cuadrado, I., \& Molero, F. (2002). Liderazgo transformacional y género: autoevaluaciones de directivos y directivas españoles [Transformational leadership and gender: Spanish managers' self-ratings]. Revista de Psicología del Trabajo y de las Organizaciones, 18, 3955.

Cuadrado, I. (2003). ¿Emplean hombres y mujeres deferentes estilos de liderazgo? Análisis de la influencia de los estilos de liderazgo en el acceso a los puestos de dirección. Revista de psicología social, 18(3), 283-307. 
Dumdum, U. R., Lowe, K. B., \& Avolio, B. J. (2002). A meta-analysis of transformational and transactional leadership correlates of effectiveness and satisfaction: An update and extension.

Dvir, T., Eden, D., Avolio, B. J., \& Shamir, B. (2002). mpact of transformational leadership on follower development and performance: A field experiment. Academy of Management Journal, 45(4), 735-744.

Druskat, V. U. (1994). Gender and leadership style: transformational and transactional leadership in the Roman Catholic Church. The Leadership Quarterly, 5(2), 99-119.

Eagly, A. H., \& Carli, L. L. (2003). The female leadership advantage: An evaluation of the evidence. The leadership quarterly, 14(6), 807-834.

Eagly, A. H., Wood, W., \& Diekman, A. B. (2000). Social role theory of sex differences and similarities: A current appraisal. The developmental social psychology of gender, 123-174.

Eagly, A. H., \& Johannesen-Schmidt, M. C. (2001). The leadership styles of women and men. Journal of social issues, 57(4), 781-797.

Eagly, A. H., Johannesen-Schmidt, M. C., \& Van Engen, M. L. (2003). Transformational, transactional, and laissez-faire leadership styles: a meta-analysis comparing women and men. Psychological bulletin, 129(4), 569.

Engen, M. L., Leeden, R., \& Willemsen, T. M. (2001). Gender, context and leadership styles: A field study. Journal of occupational and organizational psychology, 74(5), 581-598.

Enríquez, A. D. B., Martínez, X. T., Hernández, C. M., \& Reyes, M. R. (2012). Principales Estilos De Liderazgo De La Mujer Empresaria En Ciudad Valles San Luis Potosí, México. Tlatemoani, (10).

Guirado, I. C., Alonso, F. M., \& Navas, M. (2003). El liderazgo de hombres y mujeres: diferencias en estilos de liderazgo, relaciones entre estilos y predictores de variables de resultado organizacional [Leadership in men and women: Differences on leadership styles and the relationship between styles...]. Acción psicológica, 2(2), 115-129.

House, R. J. (1971). A path goal theory of leader effectiveness. Administrative science quarterly, $321-339$.

Howell, J. M., \& Avolio, B. J. (1993). Transformational leadership, transactional leadership, locus of control, and support for innovation: Key predictors of consolidated-business-unit performance. Journal of Applied Psychology, 78(6), 891.

Koontz, H., \& Weihrich, H. (1990). No Title. In La Organización Eficaz y la Cultura Organizacional. (pp. 303-309). México: Mc Graw-Hill.

Nader, M., \& Castro Solano, A. (2007). Influencia de los valores sobre los estilos de liderazgo: Un análisis según el modelo de liderazgo transformacional-transaccional de Bass. Universitas Psychologica, 6(3), 689-698. 
Núñez (2002), Modelo de Gerencia Educativa Bajo un Enfoque de Liderazgo Compartido para Mejorar la Estructura Organizacional en Escuelas Básicas. Trabajo de Grado. Universidad Pedagógica Experimental Libertador. Barquisimeto.

Omar, A. (2011). Liderazgo transformador y satisfacción laboral: el rol de la confianza en el supervisor. Liberabit, 17(2), 129-138.

Oshagbemi, T. (2004). Age influences on the leadership styles and behaviour of managers. Employee Relations, 26(1), 14-29.

Pascale, R. T. (1990). Managing on the Edge.

Pestana, F. M., Tortoza, A. J. C., Díaz, L. M. N., \& Rodríguez, E. D. C. C. (2011). Liderazgo transformacional y gestión educativa en contextos descentralizados/Leadership transformacional and educational management in decentralized contexts. Revista Actualidades Investigativas en Educación, 9(2).

Pérez Santiago, J. A., \& Camps del Valle, V. (2011). Manifestación del liderazgo transformacional en un grupo de supervisores de Puerto Rico. Revista de Psicología del Trabajo y de las Organizaciones, 27(1), 17-29.

Ramírez, J., \& Sgambatti, V. (2008). Liderazgo transformacional y transaccional en estudiantes de postgrados en gerencia del Área Metropolitana de Caracas. Visión gerencial, 7(2), 403-416.

Sarrió, M., Barberá, E., Ramos, A., \& Candela, C. (2002). El techo de cristal en la promoción profesional de las mujeres. Revista de psicología social, 17(2), 167-182.

Small, D. A., Gelfand, M., Babcock, L., \& Gettman, H. (2007). Who goes to the bargaining table? The influence of gender and framing on the initiation of negotiation. Journal of personality and social psychology, 93(4), 600.

Uribe-Rodríguez, A. F., Garrido-Pinzón, J., \& Rodríguez, A. M. (2011). Influencia del tipo de contratación en la calidad de vida laboral: manifestaciones del capitalismo organizacional. Revista Virtual Universidad Católica del Norte, 1(33), 101-116.

Van Engen, M. L., \& Willemsen, T. M. (2000). Gender and leadership styles: A review of the past decade.

Yang, Y. F. (2009). An investigation of group interaction functioning stimulated by transformational leadership on employee intrinsic and extrinsic job satisfaction. Social, Behavior and Personality, 37, 1259-1278.

Yukl, G. (2010). Why flexible and adaptive leadership is essential., 62(2), 81. 